\title{
Differential Binding of Autoantibodies to MOG Isoforms in Inflammatory Demyelinating Diseases
}

Kathrin Schanda, MSc, Patrick Peschl, PhD, Magdalena Lerch, MSc, Barbara Seebacher, PhD, Swantje Mindorf, MSc, Nora Ritter, MSc, Monika Probst, PhD, Harald Hegen, MD, PhD, Franziska Di Pauli, MD, PhD, Eva-Maria Wendel, MD, Christian Lechner, MD, Matthias Baumann, MD, Sara Mariotto, MD, PhD, Sergio Ferrari, MD, Albert Saiz, MD, PhD, Michael Farrell, MD, Maria Isabel S. Leite, MD, DPhil, Sarosh R. Irani, MD, DPhil, Jacqueline Palace, FRCP, DM, Andreas Lutterotti, MD, Tania Kümpfel, MD, Sandra Vukusic, MD, Romain Marignier, MD, PhD, Patrick Waters, PhD, Kevin Rostasy, MD, Thomas Berger, MD, Christian Probst, PhD, Romana Höftberger, MD, and Markus Reindl, PhD

Neurol Neuroimmunol Neuroinflamm 2021;8:e1027. doi:10.1212/NXI.0000000000001027

\section{Abstract}

\section{Objective}

To analyze serum immunoglobulin G ( $\mathrm{IgG}$ ) antibodies to major isoforms of myelin oligodendrocyte glycoprotein (MOG-alpha 1-3 and beta 1-3) in patients with inflammatory demyelinating diseases.

\section{Methods}

Retrospective case-control study using 378 serum samples from patients with multiple sclerosis (MS), patients with non-MS demyelinating disease, and healthy controls with MOG alpha-1IgG positive $(n=202)$ or negative serostatus $(n=176)$. Samples were analyzed for their reactivity to human, mouse, and rat MOG isoforms with and without mutations in the extracellular MOG Ig domain (MOG-ecIgD), soluble MOG-ecIgD, and myelin from multiple species using live cell-based, tissue immunofluorescence assays and ELISA.

\section{Results}

The strongest IgG reactivities were directed against the longest MOG isoforms alpha-1 (the currently used standard test for MOG-IgG) and beta-1, whereas the other isoforms were less frequently recognized. Using principal component analysis, we identified 3 different binding patterns associated with non-MS disease: (1) isolated reactivity to MOG-alpha- $1 /$ beta- $1(n=73)$, (2) binding to MOG-alpha-1/beta- 1 and at least one other alpha, but no beta isoform $(\mathrm{n}=64)$, and $(3)$ reactivity to all $6 \mathrm{MOG}$ isoforms $(\mathrm{n}=65)$. The remaining samples were negative $(\mathrm{n}=176)$ for MOG-IgG. These MOG isoform binding patterns were associated with a non-MS demyelinating disease, but there were no differences in clinical phenotypes or disease course. The 3 MOG isoform patterns had distinct immunologic characteristics such as differential binding to soluble MOG-ecIgD, sensitivity to MOG mutations, and binding to human MOG in ELISA.

\author{
Correspondence \\ Dr. Reindl \\ markus.reindl@i-med.ac.at
}




\section{Glossary}

ADEM = acute disseminated encephalomyelitis; $\mathbf{C B A}=$ cell-based assay; FACS = fluorescence-activated cell sorting; HCs = healthy controls; IF = immunofluorescence; MOG = myelin oligodendrocyte glycoprotein; MOGAD = MOG-IgG-associated disorders; MOG-ecIgD = extracellular MOG immunoglobulin domain; MOG-IgG = serum IgG antibodies against MOG; $\mathbf{M S}=$ multiple sclerosis; NMOSD = neuromyelitis optica spectrum disorder; $\mathbf{O N}=$ optic neuritis; $\mathbf{P C A}=$ principal component analysis.

\section{Conclusions}

The novel finding of differential MOG isoform binding patterns could inform future studies on the refinement of MOG-IgG assays and the pathophysiologic role of MOG-IgG.

Serum immunoglobulin G (IgG) autoantibodies against myelin oligodendrocyte glycoprotein (MOG-IgG) are associated with a spectrum of neurologic diseases including optic neuritis $(\mathrm{ON})$, acute disseminated encephalomyelitis (ADEM), myelitis, seizures, encephalitis, and with brainstem and/or cerebellar involvement. ${ }^{1-9}$ In addition, MOG-IgG appears to be supportive to discriminate these disorders from multiple sclerosis $(\mathrm{MS})^{10,11}$ as reflected by the first diagnostic recommendations for MOG-IgG-associated disorders (MOGADs). ${ }^{1,6,12}$ Furthermore, MOGADs are not only characterized by clinical but also neuropathologic features. ${ }^{13,14}$

Although most studies use live cell-based assays (CBAs) with the MOG alpha ( $\alpha$ ) 1 isoform for the measurement of MOG$\mathrm{IgG}$, previous results were often discrepant, because of different MOG expression vectors (full-length vs extracellular domain), cell lines, read-out systems (immunofluorescence [IF] vs flow cytometry), and other test variations, which aimed to increase specificity and eliminate nonspecific lowtiter positivity. ${ }^{1,15-17}$ Several studies attempted to define the molecular epitopes of MOG-IgG with the help of amino acid substitutions or deletions and discovered distinct binding patterns. ${ }^{18-21}$ The most frequent epitopes were located in the loops between the $\beta$ sheets of the extracellular Ig domain of human MOG (MOG-ecIgD). These findings were extended by other studies showing that only a subset of human MOG$\operatorname{IgG}$ is also reactive to rodent MOG epitopes ${ }^{18,19,22,23}$ and pathogenic in vitro or in vivo. ${ }^{19,23,24}$

Earlier studies using MOG-ecIgD as an antigen for immunoassays have indicated a lower sensitivity compared with fulllength MOG (with the $\alpha 1$ isoform as the consensus sequence). ${ }^{10,25}$ Although these results indicated binding differences to different MOG variants, no study so far has analyzed antibody responses to different MOG isoforms. Like most other human myelin genes, the MOG gene undergoes extensive alternative splicing and multiple different MOG isoforms have been described in primates, but not in rodents. ${ }^{26-29}$ Although the extracellular Ig domain is present in all these isoforms, they show profound differences in the composition of the intracellular $\mathrm{C}$ terminus resulting in alpha $(\alpha)$ or beta $(\beta)$ isoforms.
The aim of our study was to analyze the serum IgG antibody response to additional MOG isoforms ( $\alpha 2, \alpha 3, \beta 1, \beta 2$, and $\beta 3$; figure 1) in MOGa1-seropositive and MOGal-seronegative patients and controls. Furthermore, we analyzed whether the use of additional MOG isoforms (either alone or in combinations) improves the specificity of MOG-IgG CBAs.

\section{Methods}

\section{Patients and Controls}

We performed a retrospective case-control study including serum samples from 378 patients with inflammatory demyelinating diseases and healthy controls (HCs) with known MOG-IgG serostatus recruited from the participating centers for this study (Innsbruck and Vienna, Austria; Verona, Italy; Barcelona, Spain; Dublin, Ireland; Oxford, UK; Zurich, Switzerland; Munich and Lübeck, Germany; and Lyon, France). ${ }^{2,10,16,22}$ We included samples from children (age $<18$ years) and adults ( $\geq 18$ years) with an aquaporin-4seronegative non-MS demyelinating disease typically associated with MOG-IgG $(\mathrm{n}=214$, thereof 191 seropositive MOGAD), MS $(\mathrm{n}=64),{ }^{11}$ and HCs $(\mathrm{n}=100)$. We also included MOG-IgG-positive MS $(\mathrm{n}=8)$ and HC $(\mathrm{n}=3)$ samples collected at different centers, and therefore, MOGIgG seropositivity in MS and HCs is overrepresented compared with previous studies. Samples were sent to Innsbruck and reclassified there as MOG-IgG negative $(\mathrm{n}=176 ; 47 \%$; MOG-IgG(H $+\mathrm{L})$ titer 0 to $1: 80$; MOG-IgG $(\mathrm{Fc})$ negative $)$ or MOG-IgG positive $(\mathrm{n}=202 ; 53 \%$; MOG- $\operatorname{IgG}(\mathrm{H}+\mathrm{L})$ titer 1:160 to $1: 20,480$; MOG-IgG( $\mathrm{Fc})$ positive) using a combination of 2 different live IF MOG $\alpha 1$ CBAs (CBA-IF) as recently described. ${ }^{16}$ In addition, 106 follow-up samples of 35 individuals were analyzed with a median observation period of 4.1 years (range 0.8-12.1 years). Because a detailed clinical and radiologic description was not the primary scope of this study, we summarized clinical data of non-MS patients in predominant clinical phenotypes: opticospinal (isolated optic neuritis, myelitis, brainstem syndrome, or a combination of those, a proportion of these patients fulfilled the current diagnostic criteria for neuromyelitis optica spectrum disorder ${ }^{30}$ ), cerebral (ADEM according to current diagnostic criteria, ${ }^{31}$ 
multiphasic disseminated encephalomyelitis, brainstem encephalitis, or encephalitis), or a combination of both (ADEM followed by optic neuritis and opticospinal with cerebral symptoms) and disease courses (monophasic vs recurrent). Clinical and demographic data according to the MOG-IgG serostatus are shown in table e-1, links.lww.com/NXI/A498.

Neuropathologic investigations were available from 6 MOGIgG-positive cases: 2 with biopsy and autopsy, 3 with biopsy, and 1 with autopsy. Four of these patients had previously been described as case reports and included in a series of patients to describe the pathology of CNS demyelination accompanied by MOG-IgG. ${ }^{13}$ Serum/CSF samples and biopsy/autopsy specimens were referred to the Division of Neuropathology and Neurochemistry, Department of Neurology, Medical University of Vienna for diagnostic purposes and stored at the institutional biobank.

\section{Standard Protocol Approvals, Registrations, and Patient Consents}

This study was approved by the ethical committees of the Medical University of Innsbruck, Austria (AM3041A and AM4059), Medical University of Vienna (EK 1636/2019 and 1123/2015), University of Oxford, UK (REC 16/SC/0224),
Hospital Clinic of Barcelona, Spain (2010/5680), and University of Zürich, Switzerland (KEK ZH 2013-0001). Twenty serum samples were obtained from the NeuroBioTecHospices Civils de Lyon biobank, 10 samples from the Neuropathology-Verona biobank, and 10 samples from the Biobank of LMU Munich, Germany (Biobank 163-16). All patients or their caregivers and controls gave written informed consent. All samples from participating centers were anonymized before sending them to Innsbruck, Austria.

\section{Cloning of MOG Isoform Constructs}

The following human MOG isoforms shown in figure 1 were expressed as C-terminal enhanced green fluorescent protein fusion proteins (EGFP) in this study: MOG $\alpha 1$ (NCBI CCDS34370.1; UniProt Q16653-1), MOGa2 (NCBI CCDS47394.1; UniProt Q16653-2), MOGa3 (NCBI CCDS34369.1; UniProt Q166533), MOGß1 (NCBI CCDS4667.1; UniProt Q16653-5), MOG $\beta 2$ (NCBI CCDS34367.1; UniProt Q16653-6), and MOGß3 (NCBI CCDS34366.a; UniProt Q16653-7). The detailed cloning strategies are described in Supplementary methods. In addition, we used pEGFP-N1 (Takara Clontech) expression vectors encoding mouse MOG, rat MOG, and the human MOG 1 mutants N31Q(N-glycosylation site ${ }^{18,20,21,23,32}$ ), P42S (mouse/rat specific, immunodominant human MOG

Figure 1 Splicing Variants and Protein Isoforms of Human MOG a1-3 and $\beta 1-3$

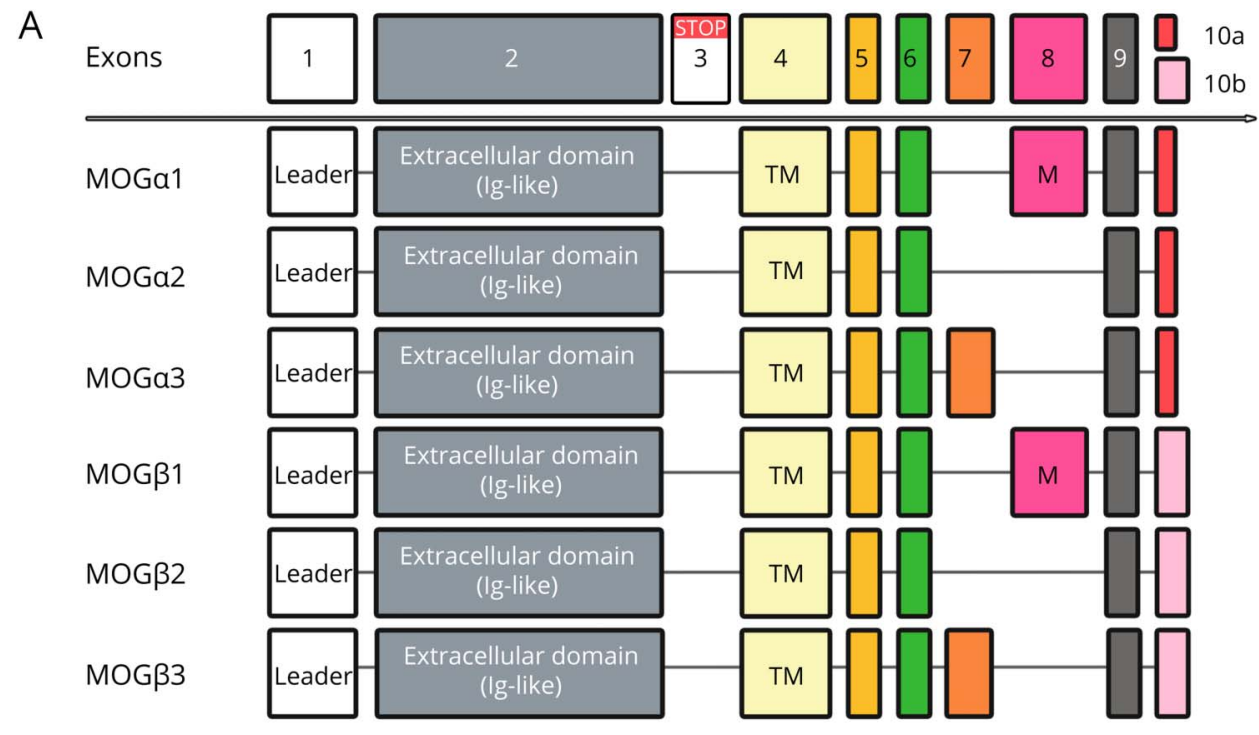

B

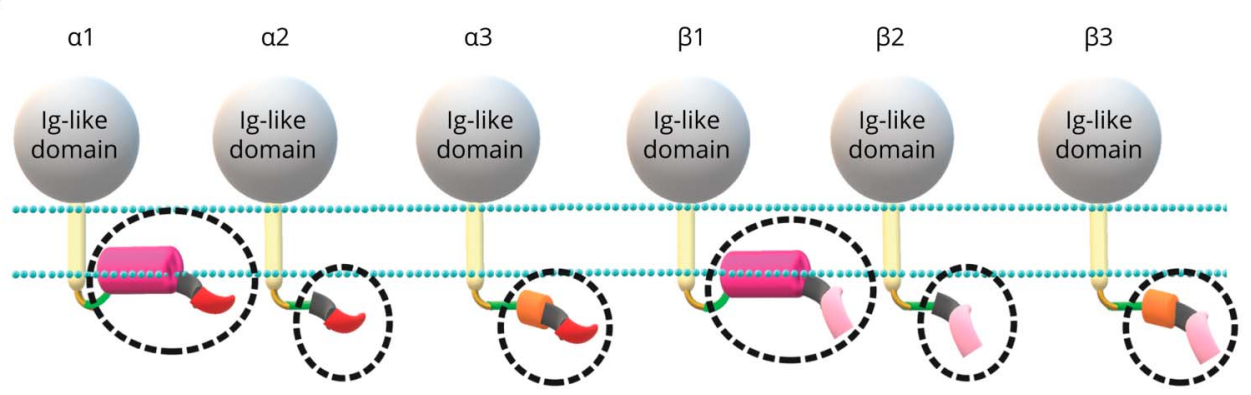

(A) Exon composition of different MOG precursor transcript variants: leader = signal peptide, removed during maturation, exon $3=$ contains a stop codon and is present in transcripts for soluble MOG (not depicted in this schematic), TM = encodes the single transmembrane domain, $\mathrm{M}$ = encodes the membrane-associated intracellular part of transcripts a 1 and $\beta 1$, exon $7=$ specific for transcripts a3 and $\beta 3$. The last $3^{\prime}$ exons encode specific sequences for MOGa1-3 (10a) and MOGß1-3 (10b). (B) Protein isoforms of human MOG $\alpha 1-3$ and $\beta 1-3$. The extracellular Ig-like domain is present in all isoforms (gray), whereas the specific intracellular composition is shown slightly enlarged within the dashed circles using colors fitting to the coding exons described above. MOG $=$ myelin oligodendrocyte glycoprotein. 
epitope ${ }^{18,21,23,32}$ ), E64K (possible binding motif for complement $\mathrm{C}_{1 \mathrm{q}^{32}}$ ), A75S (rat specific ${ }^{18,32}$ ), R86Q (mouse/rat specific $^{18,32}$ ), and H103A + S104E (epitope of the mouse monoclonal MOG antibody 8-18-C5, important human MOG epitope $\left.{ }^{18,21,23,32,33}\right)$ as described in Supplementary methods.

\section{Immunoassays for the Detection of MOG-IgG Binding to Different MOG Isoforms}

All live CBAs were performed using HEK293 cells transfected with the individual expression vectors encoding the different MOG isoforms described above. Because of the amount of analyses per sample, evaluation of antibody binding to the different isoforms and animal species was performed on the same day to reduce the number of freeze-thawing cycles. All CBAs (live CBA-IF, live CBA-fluorescence-activated cell sorting [FACS], and a commercial fixed CBA-IF from Euroimmun) and ELISAs were performed according to recently published detailed protocols. ${ }^{16}$ We used a combination of 2 assays with secondary antibodies to human $\operatorname{IgG}(\mathrm{H}+\mathrm{L})$ and confirmation by secondary antibodies to human $\operatorname{IgG}(\mathrm{Fc})$ to exclude isolated IgM reactivity. Final results were given in titer level (CBA-IF) or binding ratio (CBA-FACS). The binding of serum samples to human, mouse, and rat myelin was analyzed by a tissue-based IF assay as described before. ${ }^{19}$

\section{Competitive Binding Assay Using the Soluble Extracellular Domain of MOG}

We performed competitive binding assays by $\mathrm{CBA}^{-F A C S}{ }^{16}$ to investigate whether MOG-IgG recognizes soluble human MOG-ecIgD and to determine antibody affinities. Serum samples were diluted according to their FACS median binding ratio to standardize MOG-IgG amounts. Selection of samples was based on the MOG isoform binding pattern observed in live CBA-IF. Diluted serum samples were incubated with increasing amounts $(0,1.6,3.2$, and $9.6 \mu \mathrm{M})$ of soluble human MOG-ecIgD (provided by Euroimmun, Lübeck) ${ }^{16}$ for 1 hour at room temperature and spun down at $10,000 \mathrm{~g}$ for 5 minutes. After this preabsorption step, samples were analyzed by CBAFACS. Nonlinear regression analysis was used to analyze competitive binding and calculate IC50 values.

\section{Statistical Analysis}

The primary hypothesis of this study was that there are differential disease-specific IgG antibody responses to MOG isoforms ( $\alpha 1, \alpha 2, \alpha 3, \beta 1, \beta 2$, and $\beta 3$ ). This hypothesis was tested for antibody titers using the Friedman nonparametric test, Spearman's rank nonparametric correlational analyses, and receiver operating curve analysis. Principal component analysis (PCA) was used to classify groups of $\log 2$-transformed MOGIgG titers. This unsupervised, unbiased multivariate analysis approach was used to identify the set of variables (MOG isoforms) accounting for the greatest variation present in the data set. Loading plots were generated to visualize the combination of MOG isoforms responsible for clustering. The analyses of all secondary and other endpoints focused on estimates (common OR; positive and negative predictive values [PPV and NPV]; percentages, medians, and median differences) and $95 \% \mathrm{CI}$ or
25 th to 75 th interquartile ranges. Groups were compared using Kruskal-Wallis or $\chi^{2}$ tests. Statistical significance was defined as 2 -sided $p$ value $<0.05$ (adjusted for multiple comparisons using Bonferroni's correction). Statistical analyses were performed using IBM SPSS software (IBM SPSS Statistics; Version 26.0. Armonk, NY: IBM Corp.) or GraphPad Prism 9 (GraphPad Software, La Jolla, CA).

\section{Data Availability}

The data set used and analyzed during the current study is included in the main text or the supplementary files or is available from the corresponding author on reasonable request. Expression plasmids for the different isoforms will be deposited with Addgene.

\section{Results}

\section{Differential Antibody Binding to MOG Isoforms} $\alpha 1, \alpha 2, \alpha 3, \beta 1, \beta 2$, and $\beta 3$

Because previous studies could not consistently clarify which MOG epitopes are immunodominant, we used a different approach and compared the binding of human MOG-IgG with MOG isoforms $\alpha 1, \alpha 2, \alpha 3, \beta 1, \beta 2$, and $\beta 3$. We analyzed serum samples from 378 individuals with MOG-IgG serostatus determined using MOG $\alpha 1$ live CBA (176 negative and 202 positive) and single transfections of HEK293 cells with the other MOG isoforms. Cell surface expression of isoforms was demonstrated by performing live CBA with serial dilutions of the humanized mouse monoclonal MOG antibody 8-18-C5 showing comparable detection endpoints (figure 2, A and B). Despite small differences in transfection efficiencies, MOG isoforms showed comparable specific surface binding of humanized MOG antibody 8-18-C5 (figures e-2 and e-3, links.lww.com/NXI/ A498). Therefore, all extracellular domains of the different MOG isoforms were determined equally available for antibody binding.

Overall, the strongest antibody reactivities were directed against the longest MOG isoforms $\alpha 1$ (median titer 1:160, 25 th-75th percentile $0-640)$ and $\beta 1(1: 160,0-640)$, whereas isoforms $\alpha 2(0,0-20), \alpha 3(0,0-80), \beta 2(0,0-0)$, and $\beta 3(0$, $0-0)$ were less frequently recognized $(p<0.001)$. There were strong correlations between $\alpha 1$ and $\beta 1$ (Spearman's $\rho 0.990$ ) and between $\alpha 2, \alpha 3, \beta 2$, and $\beta 3$ (Spearman's $\rho$ 0.677-0.845), whereas the correlations between $\alpha 1 / \beta 1$ and $\alpha 2 / \alpha 3 / \beta 2 / \beta 3$ were only moderate (Spearman's $\rho$ 0.427-0.545).

In a next step, we analyzed whether the individual MOG isoforms are useful for discriminating a non-MS demyelinating disease from MS or HC. As can be seen in figure 2C, low-titer antibodies against all MOG isoforms were also present in a few cases with MS and in HCs. The positive and negative predictive values for all MOG isoforms according to titer steps are shown in table e-3, links.lww.com/NXI/A498. The cutoff values associated with a PPV (for MOG-IgG associated with a clinical MOGAD phenotype) of $95 \%$ were $\geq 1: 160$ for MOG a1 (190/214 non-MS vs $11 / 164$ controls), 
$\alpha 3(89 / 214$ non-MS vs $4 / 164$ controls), and $\beta 1(185 / 214$ non-MS vs $10 / 164$ controls), $\geq 1: 40$ for MOG $\alpha 2$ (86/214 non-MS vs $5 / 164$ controls) and $\beta 2(52 / 214$ non-MS vs $2 / 164$ controls), and $\geq 1: 80$ for MOG $\beta 3$ (58/214 non-MS vs $2 / 164$ controls).

\section{Identification of Distinct Non-MS-Associated MOG Isoform Binding Patterns}

In a next step, we investigated combinations of MOG isoforms using unsupervised PCA to generate a PCA scores plot of all samples and isoforms (figure $3 \mathrm{~A}$ ). The variables driving

Figure 2 Differential Binding of Human MOG-IgG to MOG Isoforms $\alpha 1, \alpha 2, \alpha 3, \beta 1, \beta 2$, and $\beta 3$

A

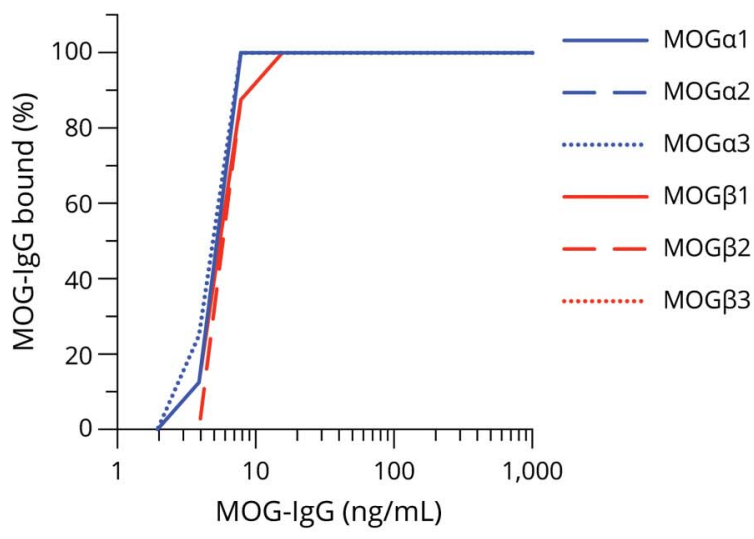

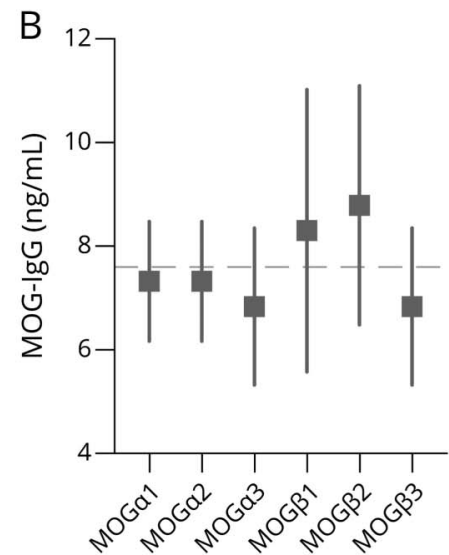

C
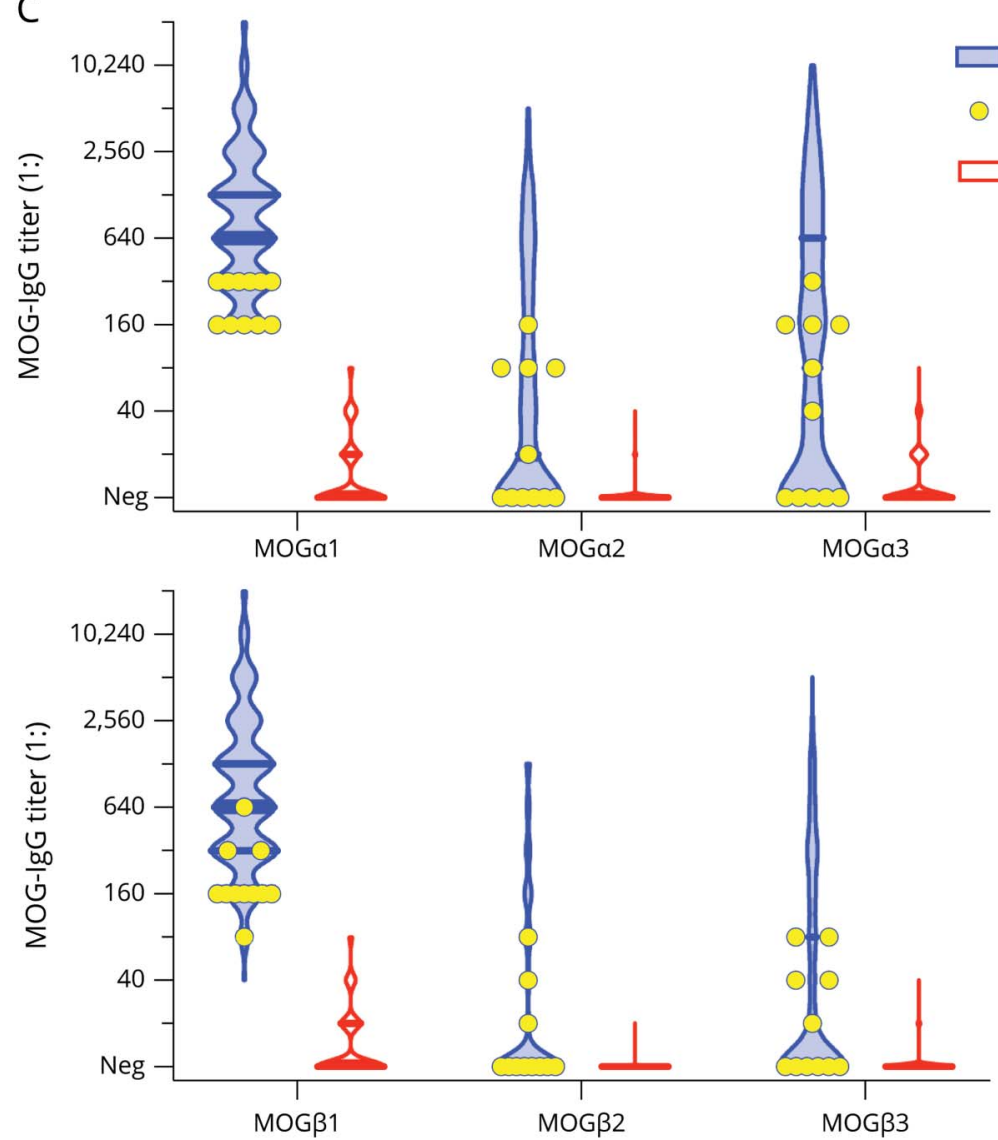

(A) Serial dilutions of humanized monoclonal mouse MOG antibody 8-18-C5. The graph shows the percentage of 8 replicates determined MOG-IgG positive in live CBA-IF transfected with individual MOG isoforms. (B) Mean sensitivity (with 95\%) for MOG-IgG in live CBA-IF using the different MOG isoforms as antigens. The dashed line indicates the overall mean sensitivity. (C) Quantitative binding of MOG-IgG to MOG isoforms a1-3 (upper panel) and $\beta 1-3$ (lower panel) for all 378 samples according to their MOG-IgG serostatus (202 positive and 176 negative). Seropositive samples with an aquaporin-4-seronegative non-MS demyelinating disease typically associated with MOG-IgG (MOGAD, $\mathrm{n}=191$ ) are shown as blue violin plots, and seropositive samples from patients with MS $(n=8)$ or healthy controls $(n=3)$ are shown as yellow circles. Seronegative samples are shown as red violin plots. Medians and interquartile ranges are indicated by the bars within violin plots. CBA = cell-based assay; HCs = healthy controls; IF = immunofluorescence; MOG = myelin oligodendrocyte glycoprotein; MOG-eclgD = extracellular MOG immunoglobulin. 


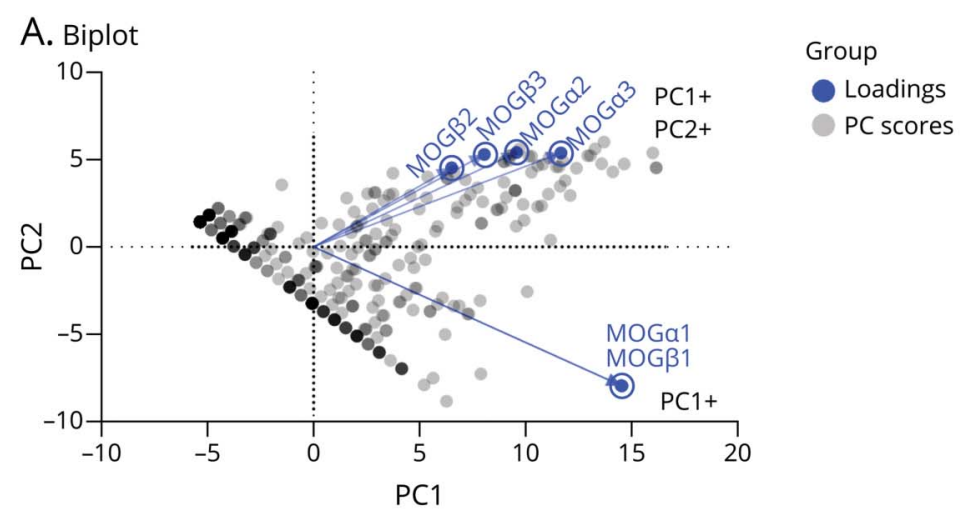

B

C
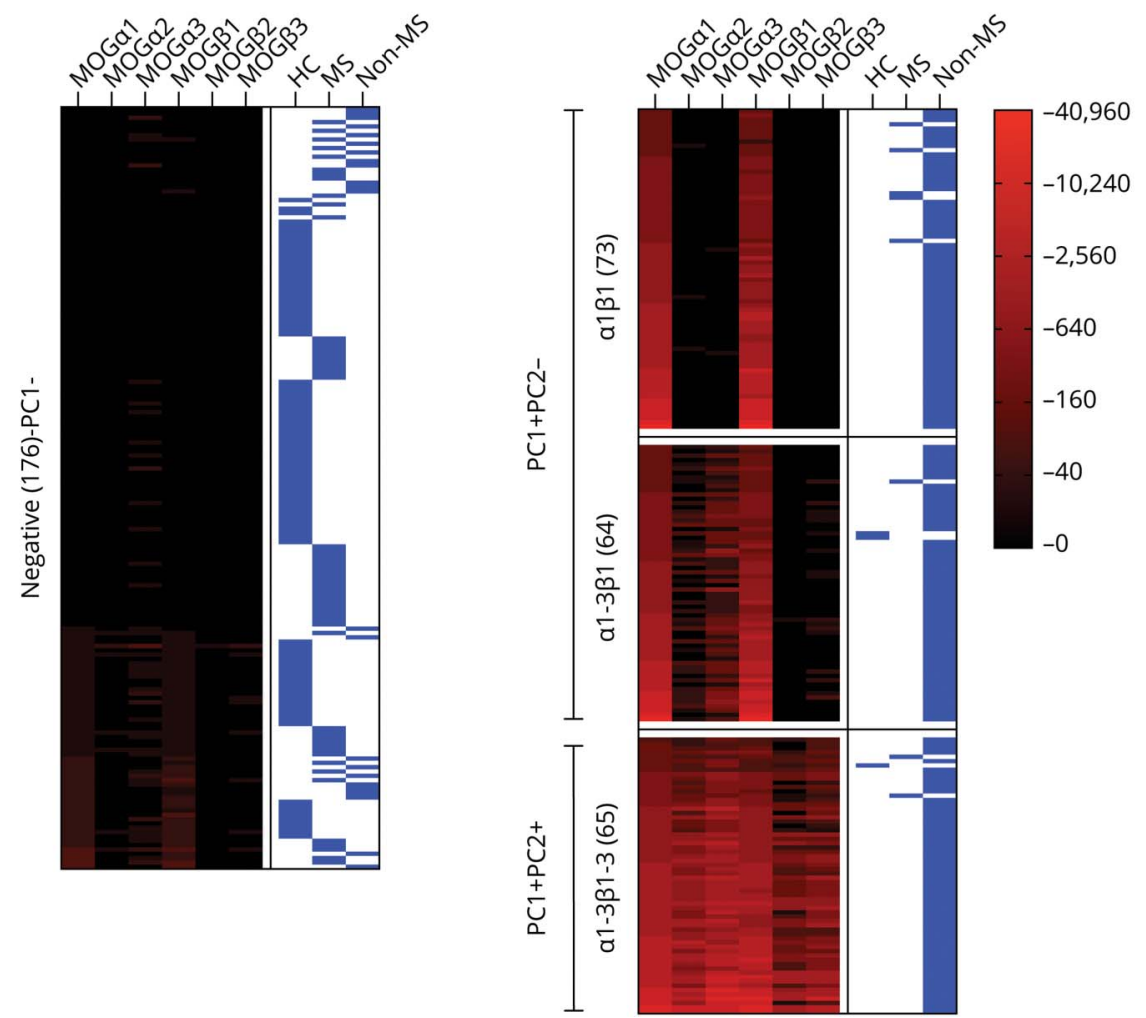

(A) Scatter dot plot showing the principal component $(\mathrm{PC})$ scores of all samples and the loading scores of all MOG isoforms to visualize parameters responsible for clustering. This identified different binding pattern clusters associated with PC1 ( $\alpha 1$ and $\beta 1)$ and/or PC2 ( $\alpha 2, \alpha 3, \beta 2$, and $\beta 3)$. Heatmap of the quantitative results (MOG-IgG titers) for all MOG-IgG-seronegative samples (B) or MOG-IgG-seropositive samples (C) according to their MOG isoform binding pattern (negative, a1 $31, \alpha 1-3 \beta 1$, and $\alpha 1-3 \beta 1-3$ ). Each column is an individual MOG isoform, and each row is an individual serum sample with MOG isoform binding patterns indicated on the left. MOG-IgG reactivities (titer 1:) are shown in different color intensities (legend with the log scale) and clinical diagnosis of non-MS, MS, and HC. (B) Individual MOG isoform IgG titers (median with the interquartile range) according to the identified binding patterns. The cutoff value for MOG-IgG positivity (a1 1:160) is indicated by the dashed blue line. (C) Percentage of patients with clinical diagnosis of non-MS (MOGAD), MS, and HC according to MOG isoform binding patterns. $\mathrm{HCs}=$ healthy controls; MOG = myelin oligodendrocyte glycoprotein: MOGAD = MOG-IgG-associated disorders; MOG$\lg \mathrm{G}=$ serum IgG antibodies against MOG.

clustering are clearly differentiated in PC1 (all isoforms with strongest contributions of $\alpha 1$ and $\beta 1 ; 75.3 \%$ of variance, Eigenvalue 28.7$)$ and PC2 $(\alpha 2, \alpha 3, \beta 2$, and $\beta 3 ; 21.2 \%$ of variance, Eigenvalue 8.1 ) together explaining $96.5 \%$ of the total variance in the data set. The biplot shown in figure $3 \mathrm{~A}$ indicates the following major groups: negative for PC1 (seronegative for MOG-IgG), positive for PC1 but negative for PC2, and positive for both PC1 and PC2. This separation into different binding clusters is shown in figure 3 and table 1 . The "negative" cluster ( $\mathrm{PC} 1$ negative, $\mathrm{n}=176)$ was associated with MOG-IgG-negative (MOG $\alpha 1$ titer 0-1:80) non-MS, MS, and $\mathrm{HC}$ samples. By contrast, the other binding patterns were predominantly associated with non-MS and dominated by either PC1 or PC2: (1) equal binding to $\alpha 1$ and $\beta 1$ and no binding to $\alpha 2, \alpha 3, \beta 2$, and $\beta 3$ (pattern $\alpha 1 \beta 1, \mathrm{n}=73$ ), (2) equal binding to $\alpha 1$ and $\beta 1$, weaker binding to $\alpha 2$ and $\alpha 3$, and no binding to $\beta 2$ and $\beta 3$ (pattern $\alpha 1-3 \beta 1, \mathrm{n}=64$ ), and (3) binding to all MOG isoforms (pattern $\alpha 1-3 \beta 1-3, \mathrm{n}=65$ ). Three representative CBA-IF stainings for samples assigned to these isoform binding patterns are shown in figure 4 .

It is important that these binding patterns were associated with a non-MS demyelinating disease consistent with MOGAD when compared with the MOG-IgG-negative patients and controls (table 1): pattern $\alpha 1 \beta 1$ (PPV 94.5\%, 95\% CI 86.7 to 97.9), pattern $\alpha 1-3 \beta 1$ (PPV 95.3\%, 95.3\% CI 87.1 to 98.7 ), and pattern $\alpha 1-3 \beta 1-3$ (PPV 93.8\%, 93.9\% CI 85.2 to 97.6). The proportion of cases with MOG $\alpha 1$ titers $\geq 1: 640$ 
Table 1 Comparison of Demographic and Clinical Data of All Patients and Controls According to Their MOG Isoform Binding Patterns

\begin{tabular}{|c|c|c|c|c|c|}
\hline & Negative $(n=176)$ & $\alpha 1 \beta 1(n=73)$ & $\alpha 1-3 \beta 1(n=64)$ & $\alpha 1-3 \beta 1-3(n=65)$ & $p$ Value $^{a}$ \\
\hline MOGa1 (1:) & $0(0-20)$ & $640(320-1,280)^{c}$ & $640(320-1,280)^{c}$ & $1,280(320-2,560)^{c}$ & $<0.001$ \\
\hline $\operatorname{MOGa2}(1:)^{b}$ & $0(0-0)$ & $0(0-0)$ & $20(0-40)^{c, d}$ & $640(160-1,280)^{\mathrm{c}, \mathrm{d}, \mathrm{e}}$ & $<0.001$ \\
\hline $\operatorname{MOGa3}(1:)^{b}$ & $0(0-0)$ & $0(0-0)$ & $160(40-160)^{c, d}$ & $1,280(640-2,560)^{\mathrm{c}, \mathrm{d}, \mathrm{e}}$ & $<0.001$ \\
\hline MOGß1 (1:) & $0(0-20)$ & $640(320-1,280)^{c}$ & $640(320-1,280)^{c}$ & $640(320-2,560)^{c}$ & $<0.001$ \\
\hline $\operatorname{MOG\beta 2~(1:)^{b}}$ & $0(0-0)$ & $0(0-0)$ & $0(0-0)$ & $160(40-320)^{c, d, e}$ & $<0.001$ \\
\hline $\operatorname{MOG\beta 3}(1:)^{b}$ & $0(0-0)$ & $0(0-0)$ & $0(0-20)$ & $320(80-640)^{c, d, e}$ & $<0.001$ \\
\hline Age $(y)^{b}$ & $36.2(18.2-45.6)$ & $17.5(7.2-42.0)^{c}$ & $18.1(6.0-36.9)^{c}$ & $23.5(8.7-43.8)$ & $<0.001$ \\
\hline Children $(<18 y)^{f, g}$ & $19.9 \%(14.7-26.4)$ & $52.1 \%(40.8-63.1)^{c}$ & $50.0 \%(38.1-61.9)^{c}$ & $40.0 \%(29.0-52.1)^{\mathrm{c}}$ & $<0.001$ \\
\hline Females $^{\mathrm{f}, \mathrm{g}}$ & $62.6 \%(54.5-70.0)$ & $46.6 \%(35.6-57.9)$ & $50.0 \%(38.1-61.9)$ & $52.3 \%(40.4-64.0)$ & ns \\
\hline \multicolumn{6}{|l|}{ Clinical diagnosis ${ }^{\mathrm{f}, \mathrm{g}}$} \\
\hline Non-MS (MOGAD) & $13.1 \%(8.9-18.8)$ & $94.5 \%(86.7-97.8)^{c}$ & $95.3 \%(87.1-89.7)^{c}$ & $93.8 \%(85.2-97.6)^{c}$ & $<0.001$ \\
\hline MS & $31.8 \%(25.4-39.0)$ & $5.5 \%(2.2-13.2)$ & $1.6 \%(0.1-8.3)$ & $4.6 \%(1.3-12.7)$ & \\
\hline $\mathrm{HC}$ & $55.1 \%(47.7-62.3)$ & $0.0 \%(0.0-5.0)$ & $3.1 \%(0.6-10.7)$ & $1.5 \%(0.1-8.2)$ & \\
\hline \multicolumn{6}{|l|}{ Clinical phenotype $e^{f, g}$} \\
\hline Cerebral $^{\mathrm{h}}$ & $56.5 \%(36.8-74.4)$ & $30.4 \%(20.9-42.1)$ & $31.1 \%(20.9-43.6)$ & $29.5 \%(19.6-41.9)$ & ns \\
\hline Opticospinal & $43.5 \%(25.6-63.2)$ & $65.2 \%(53.4-75.4)$ & $63.9 \%(51.4-74.8)$ & $62.3 \%(49.7-73.4)$ & \\
\hline Mixed $^{j}$ & $0.0 \%(0.0-14.3)$ & $4.3 \%(1.2-12.0)$ & $4.9 \%(1.3-13.5)$ & $8.2 \%(3.6-17.8)$ & \\
\hline \multicolumn{6}{|l|}{ Disease course $\mathrm{f}^{\mathrm{fg}}$} \\
\hline Monophasic & $87.0 \%(67.9-95.5)$ & $55.1 \%(43.4-66.2)$ & $49.2 \%(37.1-61.4)$ & $57.4 \%(44.9-69.0)$ & ns \\
\hline Recurrent & $13.0 \%(4.5-32.1)$ & $44.9 \%(33.8-56.6)$ & $50.8 \%(38.6-62.9)$ & $42.6 \%(31.0-55.1)$ & \\
\hline
\end{tabular}

Abbreviations: $\mathrm{ADEM}=$ acute disseminated encephalomyelitis; $\mathrm{MOG}=$ myelin oligodendrocyte glycoprotein; $\mathrm{MOGAD}=$ MOG-IgG-associated disorders; NMOSD = neuromyelitis optica spectrum disorder.

a $p$ values were adjusted for 12 comparisons using Bonferroni's correction.

${ }^{6}$ Median (interquartile range), groups were statistically compared using the Kruskal-Wallis test.

'Significantly different from the MOG-IgG-negative group.

${ }^{\mathrm{d}}$ Significantly different from the $\alpha \beta$ group.

e Significantly different from the a1-3ß1 group.

${ }^{f}$ Percentage with $95 \% \mathrm{Cl}$, groups were statistically compared using the $\chi^{2}$ test.

8 Only patients with an aquaporin-4-seronegative non-MS demyelinating disease typically associated with MOG-IgG (MOGAD).

${ }^{h}$ ADEM, MDEM, brainstem encephalitis, or encephalitis.

'Isolated optic neuritis, myelitis, brainstem syndrome, or a combination thereof (including NMOSD).

${ }^{j}$ ADEMON or opticospinal presentation with cerebral involvement.

(high-titer MOG-IgG positive) specifically associated with MOGAD $^{16}$ was higher in the $\alpha 1-3 \beta 1-3(74 \%)$ than in the $\alpha 1-$ $3 \beta 1(58 \%)$ or $\alpha 1 \beta 1(59 \%)$ binding patterns.

Furthermore, we analyzed 106 available follow-up samples of 35 cases ( $7 \alpha 1 \beta 1,15 \alpha 1-3 \beta 1$, and $13 \alpha 1-3 \beta 1-3)$ to assess the temporal stability of these binding patterns. As shown in figure e-4, links.lww.com/NXI/A498, 3 of the 7 (43\%) patients with $\alpha 1 \beta 1,10$ of the 15 (71\%) patients with $\alpha 1-3 \beta 1$, and 9 of the 13 (69\%) patients with $\alpha 1-3 \beta 1-3$ binding patterns kept their isoform binding pattern. The other patients converted to $\alpha 1 \beta 1$ (3) or $\alpha 1-3 \beta 1$ (3), or became seronegative (7). There was no conversion from $\alpha 1 \beta 1$ to $\alpha 1-3 \beta 1-3$ or vice versa.

\section{Associations of Different MOG-IgG Binding Patterns With Non-MS Clinical Phenotype, Disease Course, and Neuropathology}

Children with a non-MS disease course were more frequently associated with MOG isoform binding patterns $\alpha 1 \beta 1$ and $\alpha 1$ $3 \beta 1$, whereas adults were more frequently associated with $\alpha 1$ $3 \beta 1-3$ or seronegative MOG-IgG (table 1 ). The percentage of females was comparable between binding patterns. Furthermore, the percentage of patients with different clinical presentations of a non-MS demyelinating disease (cerebral, opticospinal, or mixed presentation) or monophasic or recurrent course at the last follow-up was comparable in all 3 MOG isoform binding patterns. Moreover, there was no association of IgG titers or seropositivity against the individual 


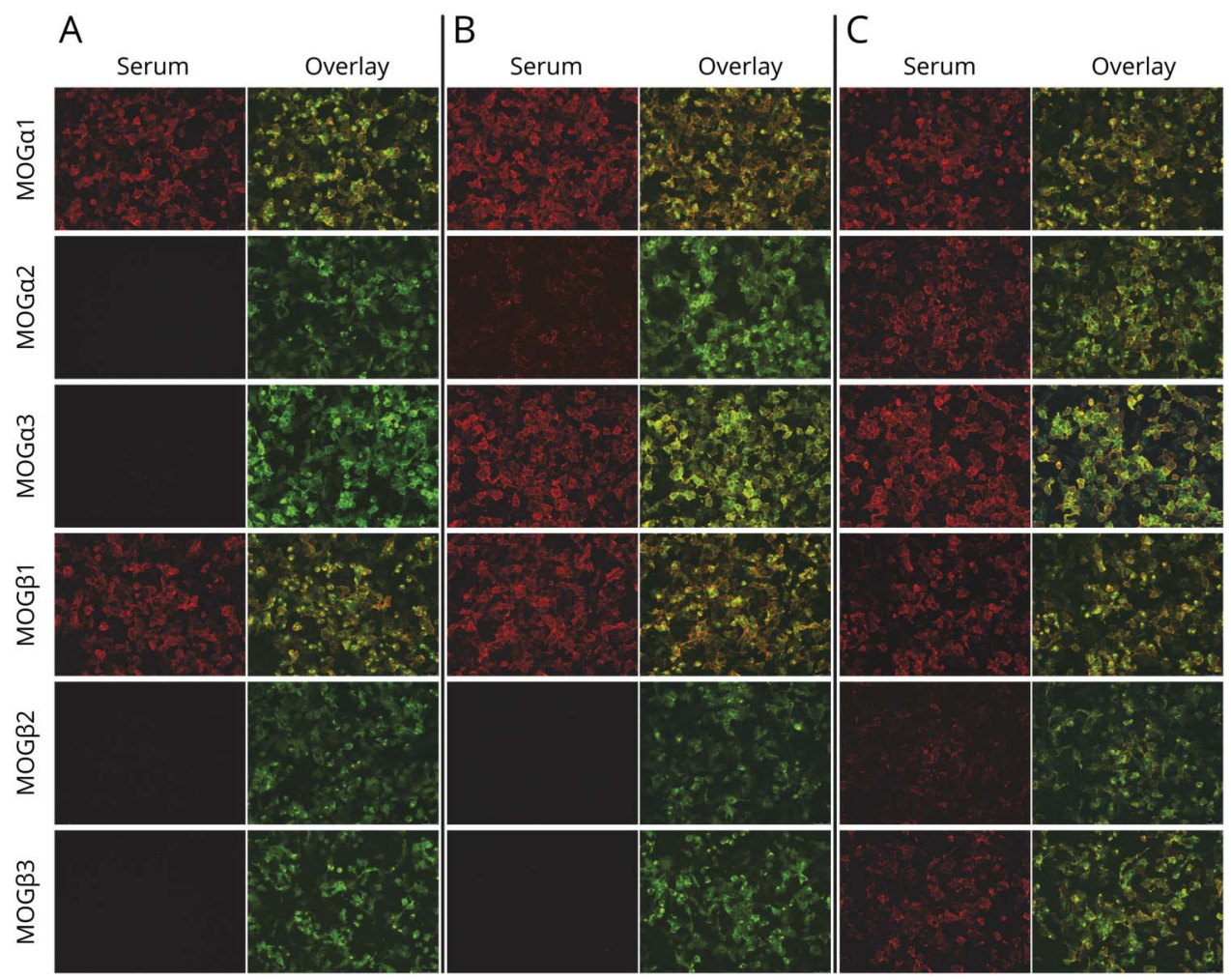

Representative live CBA-IF serum stainings for the 3 identified MOG isoform antibody binding patterns (A, a1 $\beta 1 ; B, \alpha 1-3 \beta 1 ; C, \alpha 1-3 \beta 1-3)$. Only specific antibody (red) and overlay images (MOG-transfected cells are shown in green) were used to reduce image size (20× magnification). CBA = cell-based assay; IF = immunofluorescence; $\mathrm{MOG}=$ myelin oligodendrocyte glycoprotein.

MOG isoforms with demographic or clinical parameters (tables e-4 and e-5, links.lww.com/NXI/A498).

Finally, we analyzed the neuropathologic features at disease/ relapse onset and follow-up in the 6 cases with available biopsies and/or autopsies (figure e-5, links.lww.com/NXI/ A498). One of these cases (perivenous demyelination) had $\alpha 1 \beta 1$ antibodies, 1 had the $\alpha 1-3 \beta 1$ binding pattern (perivenous demyelination, conversion to low-titer MOG-IgG at follow-up), and 4 had the $\alpha 1-3 \beta 1-3$ binding pattern ( 1 with perivenous and 3 with confluent demyelination, one of them with intrathecal MOG-IgG).

\section{MOG-IgG Binding Patterns to Different Isoforms Are Associated With a Differential Recognition of MOG Epitopes}

We performed a number of additional experiments (reactivity to several mutations in the extracellular Ig domain of MOG $\alpha 1$, competitive binding experiments with soluble MOG-ecIgD, reactivity in CBA-FACS and ELISA, reactivity to fixed MOG expressing cells, and reactivity to human, mouse, and rat MOG in brain tissue) to elucidate the molecular mechanisms underlying the 3 different MOG isoform binding patterns in a subset of samples. Figure $5 \mathrm{~A}$ shows the differential binding to human MOG $\alpha 1$ mutants, as well as mouse and rat MOG observed in the 3 MOG-IgG binding patterns in relation to MOGa1. The P42S and E64K mutations strongly affected MOG-IgG binding in all 3 binding patterns, with the strongest reduction seen in the $\alpha 1 \beta 1$ pattern. The N31Q, A75S, R86Q and H103A + S104E mutations had comparable effects in all binding patterns. Binding to mouse and rat MOG was reduced in all 3 isoform binding patterns.

Next, we analyzed whether the binding to different MOG isoforms can be inhibited by competition with increasing concentrations $(0,1.6,3.2$, and $9.6 \mu \mathrm{M})$ of soluble human MOG-ecIgD in a CBAFACS competitive binding assay. As shown in figure 5B, addition of soluble MOG-ecIgD was able to reduce the binding to MOG $\alpha 1$ in the $\alpha 1-3 \beta 1-3$ pattern comparable with the monoclonal MOG antibody 8-18-C5, but not in pattern $\alpha 1 \beta 1$. Furthermore, pattern

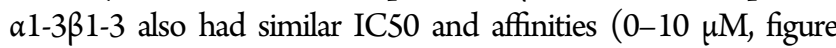
$5 C)$. By contrast, antibodies of pattern $\alpha 1 \beta 1$ did not seem to bind to the soluble MOG-ecIgD. Of interest, pattern $\alpha 1-3 \beta 1$ appears to hold a position between the 2 . Because median MOG-IgG titers were comparable (1:640 for all 3 binding patterns), we can exclude an antibody concentration effect. Similar findings were obtained using an MOG-IgG ELISA in which $\alpha 1-3 \beta 1-3$ antibodies showed better binding than the other patterns (figure 5D). By contrast, we observed no differences for reactivity in CBA-FACS, fixed CBA-IF, and antibody binding to human, mouse, or rat myelin.

\section{Discussion}

Our results show that the strongest MOG-IgG responses are directed against the longest MOG isoforms $\alpha 1$ and $\beta 1$, whereas the shorter isoforms $\alpha 2, \alpha 3, \beta 2$, and $\beta 3$ are less 


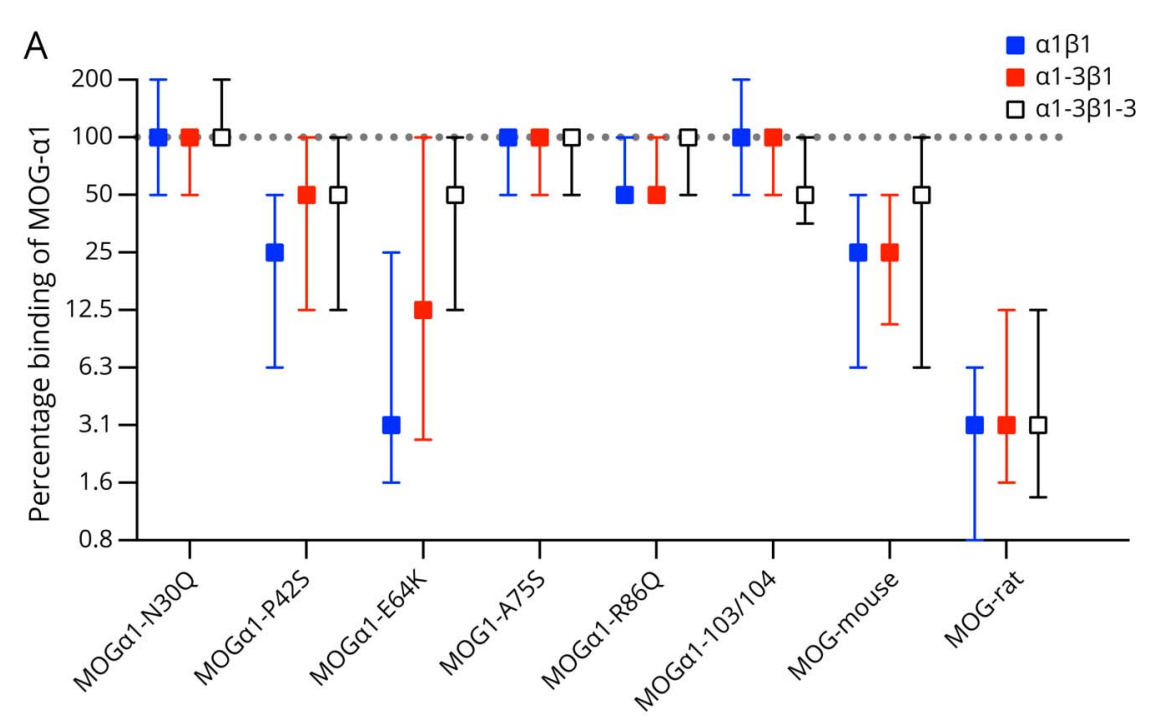

B

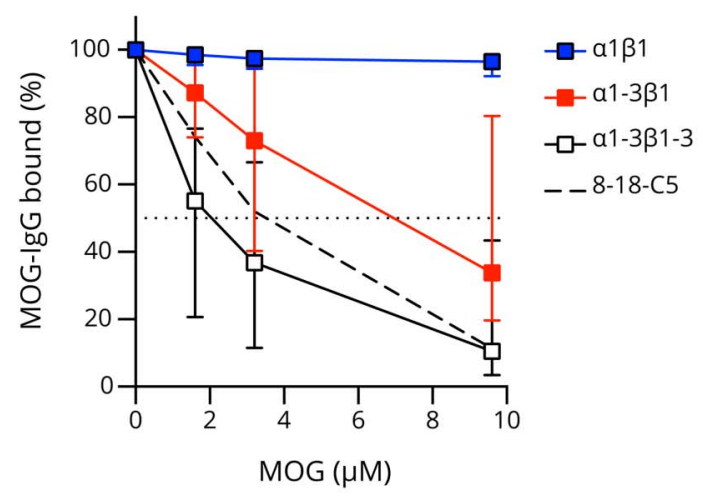

C

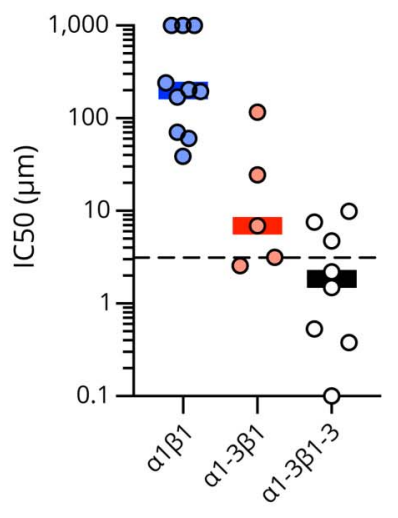

(A) Binding of MOG-IgG to the human MOGa1 mutants N31Q, P42S, E64K, A75S, R86Q, and $\mathrm{H} 103 \mathrm{~A}+\mathrm{S} 104 \mathrm{E}(103 / 104)$ and to mouse and rat MOG according to their MOG isoform binding patterns ( $\alpha 1 \beta 1, n=64 ; \alpha 1-3 \beta 1, n=43 ; \alpha 1-3 \beta 1-3$, $n=46)$. The median differences compared to wild-type MOGa1 are shown as symbols with interquartile ranges (error bars). (B) Competition of binding to MOGa1 in a CBA-FACS assay by soluble MOG-eclgD added in increasing concentrations $(0,1.6,3.2$, and $9.6 \mu \mathrm{M})$ for the $3 \mathrm{MOG}$ isoform binding patterns ( $\alpha 1 \beta 1, \mathrm{n}=10$; $\alpha 1-3 \beta 1$, $\mathrm{n}=5$; $\alpha 1-3 \beta 1-3, \mathrm{n}=8$ ) and for monoclonal antibody $8-18-C 5$ (dashed line). Squares indicate the median percentage bound, error bars indicate the interquartile ranges, and the value used for the calculation of $I_{50}(50 \%$ binding) is indicated by the dotted line. Groups were statistically compared using repeated measures 2-way analysis of variance. $(C) I C_{50}$ values for the competition of binding to MOGa1 in a CBA-FACS assay by soluble MOG-eclgD according to MOG isoform binding patterns ( $\alpha 1 \beta 1, n=10 ; \alpha 1-3 \beta 1, n$ $=5$; $a 1-3 \beta 1-3, n=8$ ). Individual data points are shown by scatter dots, and medians and interquartile ranges are indicated by lines and error bars. The $I C_{50}$ value for monoclonal antibody 8$18-C 5$ is indicated by the dashed line. (D) Binding of human MOG-IgG according to the 3 MOG isoform binding patterns to human, mouse, and rat myelin in brain sections, as well as in ELISA (human MOG Ig domain), CBA-FACS (human MOGa1), and a commercial fixed CBA-IF (human MOGX11). The percentages of myelin/MOGIgG-positive samples are shown as bars with $95 \% \mathrm{Cl}$ (error bars). CBA = cell-based assay; FACS = fluorescence-activated cell sorting; IF = immunofluorescence; $M O G=$ myelin oligodendrocyte glycoprotein; MOGAD = MOG-IgG-associated disorders; MOG-eclgD = extracellular MOG immunoglobulin domain; MOG-lgG = serum IgG antibodies against MOG.

frequently recognized. Using PCA, we could confirm 3 distinct MOG isoform binding patterns specific for patients with MOG-IgG-associated non-MS demyelinating disease (MOGAD): (1) isolated binding to MOG $\alpha 1$ and $\beta 1$ only ( $\alpha 1 \beta 1)$, (2) a mixed binding pattern with dominant recognition of $\alpha 1$ and $\beta 1$ and at least 1 additional alpha MOG isoform ( $\alpha 1-3 \beta 1$ ), and (3) binding to all MOG isoforms ( $\alpha 1-3$ $\beta 1-3)$. Recently, we demonstrated that high-titer positive MOG-IgG (MOGa1 $\geq 1: 640)$ are consistently positive in all live CBAs and specific for non-MS demyelinating disease, whereas low-titer positive MOG-IgG (MOGa1 1:160-1:320) are more frequently discordant and sometimes also found in 
patients with MS and HCs. ${ }^{16}$ This observation is also seen in our study with MOG-IgG-positive MS and HC cases found only in the low-titer positive group present in all 3 binding patterns. However, MOG-IgG seropositivity in MS and HCs in our study was overrepresented compared with previous studies. $^{1}$

The recognition of different MOG isoforms by MOG-IgG is not associated with relevant differences in clinical presentations, disease course, or neuropathology. Because our study has several limitations such as its retrospective design, the heterogenous observation period, and missing information on treatment, this needs further investigation in a prospective study. However, demographic and clinical characteristics of our study population are consistent with previous reports. ${ }^{1,2,5,6,9,12,22}$ Our results indicate that $\alpha 1 \beta 1$ and $\alpha 1-3 \beta 1$ MOG-IgG could be more common in children and $\alpha 1-3 \beta 1-3$ MOG-IgG in adults. This observation is consistent with previous findings showing a dominant expression of the isoforms MOG $\alpha 1$ and $\beta 1$ in the human brain, and especially in early development, only these 2 isoforms are detected. ${ }^{28,29}$

We used a number of additional immunologic investigations to clarify our findings. Only MOG-IgG of the $\alpha 1-3 \beta 1-3$, but not the $\alpha 1 \beta 1$, binding pattern also bound to soluble MOGecIgD with high affinity $(20 \mu \mathrm{M}-10 \mathrm{nM})$, which might explain why immunoprecipitation assays found MOG-IgG less frequently than CBAs ${ }^{25,34}$ and why attempts to isolate human MOG-IgG using affinity binding to MOG-ecIgD were only partly successful. $^{21,23}$ This result is surprising because all MOG-IgG presumably bind to epitopes in the extracellular Ig domain, and therefore, they should compete similarly with MOG-ecIgG. Additional studies are now needed to elucidate the underlying molecular mechanisms of this finding. Furthermore, the fact that only a subset of human MOG-IgG (those of MOG $\alpha 1-3 \beta 1-3$ binding pattern) was reactive in MOG ELISA may explain discrepancies found in the literature. $^{16,21,25,35}$ Finally, all 3 MOG isoform binding patterns show different sensitivities to mutations in the extracellular MOG Ig domain: whereas all patterns were sensitive to the P42S mutation associated with an immunodominant MOG epitope $\mathrm{18,21,23,32}$ and the E64K mutation in the $C^{\prime \prime} \mathrm{D}$ loop, ${ }^{36-38}$ this effect was even more pronounced in the $\alpha 1 \beta 1$ binding pattern. Because the $\alpha 1-3 \beta 1$ pattern shares many features with both of the other 2 patterns, it possibly represents a mixture of the other patterns present in polyclonal human serum samples. Further work is now necessary to define the molecular targets of antibodies associated with the identified MOG isoform binding patterns. However, it is a limitation of our study that we were not able to perform these experiments with all samples because of limited availability of samples and reagents.

Our data indicate that these antibody binding patterns are associated with clear structural differences, and therefore, it seems very likely that they represent different antibody clones. The observed MOG isoform binding patterns could be explained by the differential recognition of MOG dimers or multimers caused by clustering of MOG isoforms. ${ }^{37}$ This explanation is supported by a recent study demonstrating that the second hydrophobic domain of MOG (only present in $\alpha 1$ and $\beta 1$ ) enhanced the recognition of the extracellular part of MOG by human MOG-IgG. ${ }^{39}$ Moreover, MOG-IgG from most patients may require bivalent binding to MOG dimers, whereas a smaller subset of MOG-IgG shows monovalent binding to monomers such as the 8-18C5 monoclonal antibody.

To conclude, our novel finding of differential MOG isoform binding patterns could explain previous discrepant reports and instruct future studies to improve and refine MOG-IgG antibody assays.

\section{Study Funding}

This study was funded by a research grant from the Austrian Science Fund (FWF projects P32699, M. Reindl and R. Höftberger). M. Reindl was supported by the Guthy-Jackson Foundation (FFG Bridge 1 project Nr. 853209). S.R. Irani is supported by the Wellcome Trust (104079/Z/14/Z), BMA Research Grants-Vera Down grant (2013) and Margaret Temple (2017), Epilepsy Research UK (P1201), the Fulbright UK-US commission (MS Society research award), and the NIHR Oxford Biomedical Research Centre. The views expressed are those of the author(s) and not necessarily those of the NHS, the NIHR, or the Department of Health.

\section{Disclosure}

K. Schanda, P. Peschl, M. Lerch, and B. Seebacher report no disclosures relevant to the manuscript. S. Mindorf has received personal compensation from Euroimmun AG as an employee; N. Ritter has received personal compensation from Euroimmun AG as an employee; M. Probst has received personal compensation from Euroimmun AG as an employee of the Institute for Quality Assurance; H. Hegen has participated in meetings sponsored by, received speaker honoraria or travel funding from Bayer, Biogen, Merck, Novartis, Sanofi-Genzyme, Siemens, and Teva, and received honoraria for acting as a consultant for Biogen and Teva; F. di Pauli has participated in meetings sponsored by, received honoraria (lectures, advisory boards, and consultations) or travel funding from Bayer, Biogen, Celgene, Merck, Novartis, Sanofi-Genzyme, Roche, and Teva. Her institution has received research grants from Roche; E.-M. Wendel has no disclosure to report; C. Lechner served as a consultant for Roche; M. Baumann has no disclosure to report; S. Mariotto received support for attending scientific meetings by Merck and Euroimmun and received speaker honoraria from Biogen; S. Ferrari received support for attending scientific meetings by Shire, Sanofi-Genzyme, Roche, and Euroimmun; A. Saiz reports compensation for consulting services and speaker honoraria from Merck-Serono, Biogen-Idec, Sanofi-Aventis, Teva Pharmaceutical Industries Ltd, Novartis, Roche, and Alexion; M.I.S. Leite reported being 
involved in aquaporin-4 testing, receiving support from the National Health Service National Specialised Commissioning Group for Neuromyelitis Optica and the National Institute for Health Research Oxford Biomedical Research Centre, receiving speaking honoraria from Biogen-Idec, and receiving travel grants from Novartis; S.R. Irani is a coapplicant and receives royalties on patent application WO/ 210/046716 (UK patent no., PCT/GB2009/051441) entitled "Neurological Autoimmune Disorders." The patent has been licensed to Euroimmun AG for the development of assays for LGI1 and other VGKC-complex antibodies. He has received honoraria from UCB, Roivant, MedImmune, ADC Therapeutics, and Brain and MedLink Neurology; J. Palace is partly funded by highly specialized services to run a national congenital myasthenia service and a neuromyelitis service. She has received support for scientific meetings and honorariums for advisory work from Merck Serono, BiogenIdec, Novartis, Teva, Chugai Pharma, Bayer Schering, Alexion, Roche, Genzyme, MedImmune, EuroImmun, MedDay, Abide ARGENX, UCB, and Viela Bio and grants from Merck Serono, Novartis, Biogen-Idec, Teva, Abide, MedImmune, Bayer Schering, Genzyme, Chugai, and Alexion. She has received grants from the MS society, GuthyJackson Foundation, NIHR, Oxford Health Services Research Committee, EDEN, MRC, GMSI, John Fell and Myaware, and AMPLO for research studies; A. Lutterotti received personal fees and/or travel support for lectures and advice from Bayer, Biogen, Celgene, Genzyme, Merck, Novartis, Roche, Sanofi, and Teva, and he is a co-founder of Cellerys and co-inventor on a patent held by the University of Zurich on the use of peptide-coupled cells for the treatment of MS; T. Kümpfel has received speaker honoraria and/or personal fees for advisory boards from Bayer Healthcare, Teva Pharma, Merck, Novartis Pharma, SanofiAventis/Genzyme, Roche Pharma, and Biogen as well as grant support from Novartis and Chugai Pharma in the past; S. Vukusic has received grants, personal fees, and nonfinancial support from Biogen, personal fees from Celgene, grants and personal fees from MedDay, grants, personal fees, and nonfinancial support from Merck-Serono, grants, personal fees, and nonfinancial support from Novartis, grants, personal fees, and nonfinancial support from Roche, grants, personal fees, and nonfinancial support from SanofiGenzyme, and personal fees from Teva, all outside the submitted work; R. Marignier serves on scientific advisory boards for Viela Bio and has received funding for travel and honoraria from Biogen, Merck, Novartis, Roche, Teva, UCB, and Viela Bio; P. Waters and the University of Oxford are named inventors on patents for antibody assays and have received royalties. He has received honoraria or research funding from Biogen-Idec, Mereo BioPharma, Retrogenix, and Euroimmun AG, and travel grants from the GuthyJackson Charitable Foundation; K. Rostasy has served as a consultant for the PARADIGM Study/Novartis without payment; T. Berger has participated in meetings sponsored by and received honoraria (lectures, advisory boards, and consultations) from pharmaceutical companies marketing treatments for MS: Almirall, Bayer, Biogen, Biologix, Bionorica, Genzyme, MedDay, Merck, Novartis, Octapharma, Roche, Sanofi/Genzyme, TG Pharmaceuticals, TEVAratiopharm, and UCB. His institution has received financial support in the past 12 months by unrestricted research grants (Biogen, Bayer, Merck, Novartis, Sanofi/Genzyme, and TEVA ratiopharm) and for participation in clinical trials in MS sponsored by Alexion, Bayer, Biogen, Merck, Novartis, Octapharma, Roche, Sanofi/Genzyme, and TEVA; C. Probst has received personal compensation from Euroimmun AG as an employee; R. Höftberger received speaker's honoraria from Novartis and Biogen. The Medical University of Vienna (Austria; employer of Dr. Höftberger) receives payment for antibody assays (MOG, aquaporin-4, and other autoantibodies) and for MOG and aquaporin-4 antibody validation experiments organized by Euroimmun (Lübeck, Germany); M. Reindl was supported by a research support from Euroimmun and Roche. The University Hospital and Medical University of Innsbruck (Austria; employer of Dr. Reindl) receives payments for antibody assays (MOG, aquaporin-4, and other autoantibodies) and for MOG and aquaporin-4 antibody validation experiments organized by Euroimmun (Lübeck, Germany). Go to Neurology.org/ $\mathrm{NN}$ for full disclosures.

\section{Publication History}

Received by Neurology: Neuroimmunology \& Neuroinflammation January 4, 2021. Accepted in final form April 15, 2021.

\section{Appendix Authors}

\begin{tabular}{lll}
\hline Name & Location & Contribution \\
\hline $\begin{array}{l}\text { Kathrin } \\
\text { Schanda, MSc }\end{array}$ & $\begin{array}{l}\text { Medical University of } \\
\text { Innsbruck, Austria }\end{array}$ & $\begin{array}{l}\text { Drafting/revision of the } \\
\text { manuscript for content, } \\
\text { including medical writing for } \\
\text { content; major role in the } \\
\text { acquisition of data; study } \\
\text { concept or design; and analysis } \\
\end{array}$ \\
& or interpretation of data
\end{tabular}

\begin{tabular}{lll}
\hline Patrick & Medical University of & Drafting/revision of the \\
Peschl, PhD & Innsbruck, Austria & $\begin{array}{l}\text { manuscript for content, } \\
\text { including medical writing for } \\
\text { content; major role in the } \\
\end{array}$ \\
& $\begin{array}{l}\text { acquisition of data; and } \\
\text { analysis or interpretation of } \\
\text { data }\end{array}$ \\
\end{tabular}

\begin{tabular}{lll}
\hline $\begin{array}{l}\text { Magdalena } \\
\text { Lerch, MSc }\end{array}$ & Medical University of & $\begin{array}{l}\text { Drafting/revision of the } \\
\text { Innsbruck, Austria }\end{array}$ \\
& & $\begin{array}{l}\text { including medical writing for } \\
\text { content; and analysis or } \\
\text { interpretation of data }\end{array}$ \\
& &
\end{tabular}

\begin{tabular}{lll}
\hline $\begin{array}{l}\text { Barbara } \\
\text { Seebacher, }\end{array}$ & Medical University of & Drafting/revision of the \\
PhD & & $\begin{array}{l}\text { manuscript for content, } \\
\text { including medical writing for } \\
\text { content; and analysis or } \\
\text { interpretation of data }\end{array}$ \\
& & \\
\hline
\end{tabular}

\begin{tabular}{lll}
\hline Swantje & Euroimmun AG, & Drafting/revision of the \\
Mindorf, MSc & Germany & manuscript for content, \\
& including medical writing for \\
& content; and analysis or \\
& interpretation of data
\end{tabular}


Appendix (continued)

\begin{tabular}{lll}
\hline Name & Location & Contribution \\
\hline $\begin{array}{l}\text { Nora Ritter, } \\
\text { MSc }\end{array}$ & $\begin{array}{l}\text { Euroimmun AG, } \\
\text { Germany }\end{array}$ & $\begin{array}{l}\text { Drafting/revision of the } \\
\text { manuscript for content, including } \\
\text { medical writing for content; and } \\
\text { analysis or interpretation of data }\end{array}$ \\
\hline $\begin{array}{l}\text { Monika } \\
\text { Probst, PhD }\end{array}$ & $\begin{array}{l}\text { Institute for Quality } \\
\text { Assurance, Germany }\end{array}$ & $\begin{array}{l}\text { Drafting/revision of the } \\
\text { manuscript for content, } \\
\text { including medical writing for } \\
\text { content; and analysis or } \\
\text { interpretation of data }\end{array}$ \\
\hline $\begin{array}{l}\text { Harald } \\
\text { Hegen, MD, } \\
\text { PhD }\end{array}$ & Medical University of & $\begin{array}{l}\text { Drafting/revision of the } \\
\text { manuscript for content, } \\
\text { including medical writing for } \\
\text { content; and analysis or }\end{array}$ \\
& & $\begin{array}{l}\text { interpretation of data } \\
\text { instria }\end{array}$
\end{tabular}

Franziska Di Medical University of

Pauli, MD, Innsbruck, Austria

PhD

Drafting/revision of the manuscript for content including medical writing for content; and analysis or interpretation of data

\begin{tabular}{ll}
\hline Eva-Maria & Olgahospital/Klinikum \\
Wendel, MD & Stuttgart, Stuttgart, \\
& Germany
\end{tabular}

\section{Drafting/revision of the} manuscript for content, including medical writing for content; and analysis or interpretation of data

\begin{tabular}{lll}
\hline $\begin{array}{l}\text { Christian } \\
\text { Lechner, MD }\end{array}$ & $\begin{array}{l}\text { Medical University of } \\
\text { Innsbruck, Austria }\end{array}$ & $\begin{array}{l}\text { Drafting/revision of the } \\
\text { manuscript for content, } \\
\text { including medical writing for } \\
\text { content; and analysis or } \\
\text { interpretation of data }\end{array}$ \\
& &
\end{tabular}

\begin{tabular}{lll}
\hline Matthias & Medical University of & Drafting/revision of the \\
Baumann, & Innsbruck, Austria & $\begin{array}{l}\text { manuscript for content, } \\
\text { including medical writing for } \\
\text { MD }\end{array}$ \\
& $\begin{array}{l}\text { content; and analysis or } \\
\text { interpretation of data }\end{array}$
\end{tabular}

\begin{tabular}{lll}
\hline $\begin{array}{l}\text { Sara } \\
\text { Mariotto, } \\
\text { MD.PhD }\end{array}$ & $\begin{array}{l}\text { University of Verona, } \\
\text { Verona, Italy }\end{array}$ & $\begin{array}{l}\text { Drafting/revision of the } \\
\text { manuscript for content, } \\
\text { including medical writing for } \\
\text { content; and analysis or } \\
\text { interpretation of data }\end{array}$ \\
\hline $\begin{array}{l}\text { Sergio } \\
\text { Ferrari, MD }\end{array}$ & $\begin{array}{l}\text { University of Verona, } \\
\text { Verona, Italy }\end{array}$ & $\begin{array}{l}\text { Drafting/revision of the } \\
\text { manuscript for content, } \\
\text { including medical writing for } \\
\text { content; and analysis or } \\
\text { interpretation of data }\end{array}$ \\
\hline
\end{tabular}

\begin{tabular}{lll}
\hline Albert Saiz, & University of Barcelona, & Drafting/revision of the \\
MD, PhD & Spain & manuscript for content, \\
& including medical writing for \\
& content; and analysis or \\
& interpretation of data
\end{tabular}

\begin{tabular}{ll}
\hline Michael & Beaumont Hospital, \\
Farrell, MD & Dublin, Ireland
\end{tabular}

Drafting/revision of the manuscript for content including medical writing for content; and analysis or interpretation of data

Maria Isabel University of Oxford, Drafting/revision of the S. Leite, MD, Oxford, UK manuscript for content, DPhil including medical writing for content; and analysis or interpretation of data

\begin{tabular}{lll}
\hline Sarosh R. & University of Oxford, & Drafting/revision of the \\
Irani, MD, & Oxford, UK & $\begin{array}{l}\text { manuscript for content, } \\
\text { including medical writing for } \\
\text { DPhil }\end{array}$ \\
& & $\begin{array}{l}\text { content; and analysis or } \\
\text { interpretation of data }\end{array}$ \\
\hline
\end{tabular}

Appendix (continued)

\begin{tabular}{lll}
\hline Name & Location & Contribution \\
\hline $\begin{array}{l}\text { Jacqueline } \\
\text { Palace, FRCP, } \\
\text { DM }\end{array}$ & $\begin{array}{l}\text { University of Oxford, } \\
\text { Oxford, UK }\end{array}$ & $\begin{array}{l}\text { Drafting/revision of the } \\
\text { manuscript for content, } \\
\text { including medical writing for } \\
\text { content; and analysis or } \\
\text { interpretation of data }\end{array}$ \\
\hline $\begin{array}{l}\text { Andreas } \\
\text { Lutterotti, } \\
\text { MD }\end{array}$ & Zurich, Switzerland & $\begin{array}{l}\text { Drafting/revision of the } \\
\text { manuscript for content, } \\
\text { including medical writing for } \\
\text { content; and analysis or } \\
\text { interpretation of data }\end{array}$ \\
\hline
\end{tabular}

Tania Ludwig-Maximilians Drafting/revision of the

Kümpfel, MD University, Munich, manuscript for content, Germany including medical writing for content; and analysis or interpretation of data

\begin{tabular}{lll}
\hline $\begin{array}{l}\text { Sandra } \\
\text { Vukusic, MD }\end{array}$ & $\begin{array}{l}\text { Hôpital neurologique } \\
\text { Pierre Wertheimer, } \\
\text { Lyon, France }\end{array}$ & $\begin{array}{l}\text { Drafting/revision of the } \\
\text { manuscript for content, } \\
\text { including medical writing for } \\
\text { content; and analysis or } \\
\text { interpretation of data }\end{array}$ \\
\hline $\begin{array}{l}\text { Romain } \\
\text { Marignier, } \\
\text { MD, PhD }\end{array}$ & $\begin{array}{l}\text { Hôpital neurologique } \\
\text { Pierre Wertheimer, }\end{array}$ & $\begin{array}{l}\text { Drafting/revision of the } \\
\text { manuscript for content, } \\
\text { including medical writing for } \\
\text { content; and analysis or } \\
\text { interpretation of data }\end{array}$
\end{tabular}

\begin{tabular}{lll}
\hline Patrick & University of Oxford, & Drafting/revision of the \\
Waters, PhD & Oxford, UK & manuscript for content,
\end{tabular} including medical writing for content; and analysis or interpretation of data

\begin{tabular}{lll}
\hline $\begin{array}{l}\text { Kevin } \\
\text { Rostasy, MD }\end{array}$ & $\begin{array}{l}\text { Witten/Herdecke } \\
\text { University, Germany }\end{array}$ & $\begin{array}{l}\text { Drafting/revision of the } \\
\text { manuscript for content, } \\
\text { including medical writing for } \\
\text { content; and analysis or } \\
\text { interpretation of data }\end{array}$ \\
$\begin{array}{l}\text { Thomas } \\
\text { Berger, MD }\end{array}$ & $\begin{array}{l}\text { Medical University of } \\
\text { Vienna, Austria }\end{array}$ & $\begin{array}{l}\text { Drafting/revision of the } \\
\text { manuscript for content, including } \\
\text { medical writing for content; and } \\
\text { analysis or interpretation of data }\end{array}$ \\
\hline
\end{tabular}

Christian Euroimmun, Germany Drafting/revision of the manuscript
Probst, PhD for content, including medical writing for content; major role in the acquisition of data; and analysis or interpretation of data

\begin{tabular}{lll}
\hline $\begin{array}{l}\text { Romana } \\
\text { Höftberger, }\end{array}$ & Medical University of & Drafting/revision of the \\
MD & & manuscript for content, \\
& including medical writing for \\
& content; major role in the \\
& acquisition of data; study \\
& concept or design; and analysis \\
& or interpretation of data
\end{tabular}

\begin{tabular}{lll}
\hline Markus & Medical University of & Drafting/revision of the \\
Reindl, PhD & Innsbruck, Austria & manuscript for content, \\
& including medical writing for \\
& content; major role in the \\
& acquisition of data; study \\
& concept or design; and analysis \\
& or interpretation of data
\end{tabular}

\section{References}

1. Reindl M, Waters P. Myelin oligodendrocyte glycoprotein antibodies in neurological disease. Nat Rev Neurol. 2019;15(2):89-102.

2. Cobo-Calvo A, Ruiz A, Maillart E, et al. Clinical spectrum and prognostic value of CNS MOG autoimmunity in adults: the MOGADOR study. Neurology. 2018;90(21): e1858-e1869. 
3. Jurynczyk M, Messina S, Woodhall MR, et al. Clinical presentation and prognosis in MOG-antibody disease: a UK study. Brain. 2017;140(12):3128-3138.

4. Hennes EM, Baumann M, Schanda K, et al. Prognostic relevance of MOG antibodies in children with an acquired demyelinating syndrome. Neurology. 2017;89(9): 900-908.

5. Hacohen Y, Wong YY, Lechner C, et al. Disease course and treatment responses in children with relapsing myelin oligodendrocyte glycoprotein antibody-associated disease. JAMA Neurol. 2018;75(4):478-487.

6. Lopez-Chiriboga AS, Majed M, Fryer J, et al. Association of MOG-IgG serostatus with relapse after acute disseminated encephalomyelitis and proposed diagnostic criteria for MOG-IgG-associated disorders. JAMA Neurol. 2018;75(11):1355-1363.

7. Sato DK, Callegaro D, Lana-Peixoto MA, et al. Distinction between MOG antibody-positive and AQP4 antibody-positive NMO spectrum disorders. Neurology. 2014;82(6):474-481.

8. Kim SM, Woodhall MR, Kim JS, et al. Antibodies to MOG in adults with inflammatory demyelinating disease of the CNS. Neurol Neuroimmunol Neuroinflamm. 2015;2(6):e163.

9. Waters P, Fadda G, Woodhall M, et al. Serial anti-myelin oligodendrocyte glycoprotein antibody analyses and outcomes in children with demyelinating syndromes. JAMA Neurol. 2020;77(1):82-93.

10. Waters $\mathrm{P}$, Woodhall M, O'Connor KC, et al. MOG cell-based assay detects non-MS patients with inflammatory neurologic disease. Neurol Neuroimmunol Neuroinflamm. 2015;2(3):e89.

11. Thompson AJ, Banwell BL, Barkhof F, et al. Diagnosis of multiple sclerosis: 2017 revisions of the McDonald criteria. Lancet Neurol. 2018;17(2):162-173.

12. Jarius S, Paul F, Aktas O, et al. MOG encephalomyelitis: international recommendations on diagnosis and antibody testing. J Neuroinflammation. 2018;15(1):134.

13. Höftberger R, Guo Y, Flanagan EP, et al. The pathology of central nervous system inflammatory demyelinating disease accompanying myelin oligodendrocyte glycoprotein autoantibody. Acta Neuropathol. 2020;139(5):875-892.

14. Takai Y, Misu T, Kaneko K, et al. Myelin oligodendrocyte glycoprotein antibodyassociated disease: an immunopathological study. Brain. 2020;143(5):1431-1446.

15. Waters PJ, Komorowski L, Woodhall M, et al. A multicenter comparison of MOG-IgG cell-based assays. Neurology. 2019;92(11):e1250-e1255.

16. Reindl M, Schanda K, Woodhall M, et al. International multicenter examination of MOG antibody assays. Neurol Neuroimmunol Neuroinflamm. 2020;7(2):e674.

17. Gastaldi M, Scaranzin S, Jarius S, et al. Cell-based assays for the detection of MOG antibodies: a comparative study. J Neurol. 2020;267(12):3555-3564.

18. Mayer MC, Breithaupt C, Reindl M, et al. Distinction and temporal stability of conformational epitopes on myelin oligodendrocyte glycoprotein recognized by patients with different inflammatory central nervous system diseases. J Immunol. 2013;191(7):3594-3604.

19. Peschl P, Schanda K, Zeka B, et al. Human antibodies against the myelin oligodendrocyte glycoprotein can cause complement-dependent demyelination. J Neuroinflammation. 2017;14(1):208.

20. Marti Fernandez I, Macrini C, Krumbholz M, et al. The glycosylation site of myelin oligodendrocyte glycoprotein affects autoantibody recognition in a large proportion of patients. Front Immunol. 2019;10:1189.

21. Tea F, Lopez JA, Ramanathan S, et al. Characterization of the human myelin oligodendrocyte glycoprotein antibody response in demyelination. Acta Neuropathol Commun. 2019;7(1):145.
22. Sepúlveda M, Armangue T, Martinez-Hernandez E, et al. Clinical spectrum associated with MOG autoimmunity in adults: significance of sharing rodent MOG epitopes. J Neurol. 2016;263(7):1349-1360.

23. Spadaro M, Winklmeier S, Beltrán E, et al. Pathogenicity of human antibodies against myelin oligodendrocyte glycoprotein. Ann Neurol. 2018;84(2):315-328.

24. Saadoun S, Waters P, Owens GP, Bennett JL, Vincent A, Papadopoulos MC. Neuromyelitis optica MOG-IgG causes reversible lesions in mouse brain. Acta Neuropathol Commun. 2014;2:35.

25. O'Connor KC, McLaughlin KA, De Jager PL, et al. Self-antigen tetramers discriminate between myelin autoantibodies to native or denatured protein. Nat Med. 2007;13(2): 211-217.

26. Boyle LH, Traherne JA, Plotnek G, Ward R, Trowsdale J. Splice variation in the cytoplasmic domains of myelin oligodendrocyte glycoprotein affects its cellular localisation and transport. J Neurochem. 2007;102(6):1853-1862.

27. Delarasse C, Della Gaspera B, Lu CW, et al. Complex alternative splicing of the myelin oligodendrocyte glycoprotein gene is unique to human and non-human primates. J Neurochem. 2006;98(6):1707-1717.

28. Ballenthin PA, Gardinier MV. Myelin/oligodendrocyte glycoprotein is alternatively spliced in humans but not mice. J Neurosci Res. 1996;46(2):271-281.

29. Allamargot C, Gardinier MV. Alternative isoforms of myelin/oligodendrocyte glycoprotein with variable cytoplasmic domains are expressed in human brain. J Neurochem. 2007;101(2):298-312.

30. Wingerchuk DM, Banwell B, Bennett JL, et al. International consensus diagnostic criteria for neuromyelitis optica spectrum disorders. Neurology. 2015;85(2):177-189.

31. Krupp LB, Tardieu M, Amato MP, et al. International Pediatric Multiple Sclerosi Study Group criteria for pediatric multiple sclerosis and immune-mediated central nervous system demyelinating disorders: revisions to the 2007 definitions. Mult Scler. 2013;19(10):1261-1267.

32. Breithaupt C, Schubart A, Zander H, et al. Structural insights into the antigenicity of myelin oligodendrocyte glycoprotein. Proc Natl Acad Sci USA. 2003;100(16): 9446-9451.

33. Linington C, Bradl M, Lassmann H, Brunner C, Vass K. Augmentation of demyelination in rat acute allergic encephalomyelitis by circulating mouse monoclon antibodies directed against a myelin/oligodendrocyte glycoprotein. Am J Pathol. 1988;130(3):443-454.

34. Lampasona V, Franciotta D, Furlan R, et al. Similar low frequency of anti-MOG IgG and IgM in MS patients and healthy subjects. Neurology. 2004;62(11):2092-2094.

35. Di Pauli F, Mader S, Rostasy K, et al. Temporal dynamics of anti-MOG antibodies in CNS demyelinating diseases. Clin Immunol. 2011;138(3):247-254.

36. Johns TG, Bernard CC. Binding of complement component Clq to myelin oligodendrocyte glycoprotein: a novel mechanism for regulating CNS inflammation. Mol Immunol. 1997;34(1):33-38.

37. Clements CS, Reid HH, Beddoe T, et al. The crystal structure of myelin oligodendrocyte glycoprotein, a key autoantigen in multiple sclerosis. Proc Natl Acad Sci USA. 2003;100(19):11059-11064.

38. Hjelmström P, Penzotti JE, Henne RM, Lybrand TP. A molecular model of myelin oligodendrocyte glycoprotein. J Neurochem. 1998;71(4):1742-1749.

39. Macrini C, Gerhards R, Winklmeier S, et al. Features of MOG required for recognition by patients with MOG antibody-associated disorders. Brain. 2021.Epub March 11. 


\title{
Neurology \\ Neuroimmunology \& Neuroinflammation
}

\author{
Differential Binding of Autoantibodies to MOG Isoforms in Inflammatory \\ Demyelinating Diseases \\ Kathrin Schanda, Patrick Peschl, Magdalena Lerch, et al. \\ Neurol Neuroimmunol Neuroinflamm 2021;8; \\ DOI 10.1212/NXI.0000000000001027
}

This information is current as of June 15, 2021

\section{Updated Information \& \\ Services}

References

Citations

Subspecialty Collections

Permissions \& Licensing

Reprints including high resolution figures, can be found at:

http://nn.neurology.org/content/8/5/e1027.full.html

This article cites 38 articles, 6 of which you can access for free at: http://nn.neurology.org/content/8/5/e1027.full.html\#\#ref-list-1

This article has been cited by 2 HighWire-hosted articles: http://nn.neurology.org/content/8/5/e1027.full.html\#\#otherarticles

This article, along with others on similar topics, appears in the following collection(s):

All Demyelinating disease (CNS)

http://nn.neurology.org//cgi/collection/all_demyelinating_disease_cns Autoimmune diseases

http://nn.neurology.org//cgi/collection/autoimmune_diseases

Information about reproducing this article in parts (figures,tables) or in its entirety can be found online at:

http://nn.neurology.org/misc/about.xhtml\#permissions

Information about ordering reprints can be found online:

http://nn.neurology.org/misc/addir.xhtml\#reprintsus

Neurol Neuroimmunol Neuroinflamm is an official journal of the American Academy of Neurology.

Published since April 2014, it is an open-access, online-only, continuous publication journal. Copyright

Copyright (C) 2021 The Author(s). Published by Wolters Kluwer Health, Inc. on behalf of the American

Academy of Neurology.. All rights reserved. Online ISSN: 2332-7812.

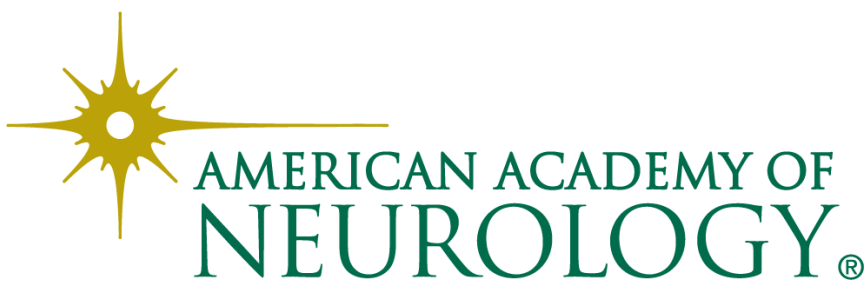

